\title{
Using Multivariate Statistics on Detection of Particular Signals during Production of Knitwear
}

\author{
A. Catarino ${ }^{1}$, A. Rocha $^{2}$, J.L. Monteiro ${ }^{3}$, F. Soares ${ }^{4}$ \\ University of Minho, Portugal \\ 1'whiteman@det.uminho.pt, ${ }^{2}$ amrocha@det.uminho.pt, ${ }^{3}$ Joao.monteiro@dei.uminho.pt, ${ }^{4}$ fsoares@dei.uminho.pt
}

\begin{abstract}
This paper reports the recent developments in the pursuit to correctly locate, identify and distinguish faults during production of weft knitted fabrics. For this purpose a major textile parameter - yarn input tension (YIT) - is analyzed by means of signal processing techniques. An overview of the entire process of gathering the information and fault detection is presented. For the purpose of distinguishing faults, Multivariate statistical methods, namely cluster and discriminant analysis are used, results presented and discussed. Finally, some conclusions are drawn from the obtained results and future developments are addressed.
\end{abstract}

\section{INTRODUCTION}

One fundamental issue concerning modern production, in which is obviously included the textile industry, is quality. It is already assumed that productivity is (or should have been) maximized. However, when it comes to make a product, all the production chain is directly involved in the quality issue. The machines have to be adequately monitored and periodic maintenance procedure is required. This constitutes a well known behavior in order to avoid as much as possible the so called non conformities in the product [1]. Nevertheless, the process of making something always involves some uncertainty, some variability between each single product, even if one is producing the very same item. Again, this behavior is explained through the intrinsic random variability during the process. When the process variability does not follow a normal distribution, then the former will be out of control and corrective measures need to be taken.

This particularity is especially true on the textile business, and for the present work, on the weft knitting production. The particular physical properties of the textiles, such as material flexibility and rigidity, perform a crucial role in the quality of the final product. In fact, textiles are much more sensible to non conformities, resulting unavoidably in faults. The measures to prevent those problems are numerous and start on the very beginning, at the raw material up to the final product $[2,3]$. The raw material itself involves already a significant variability, thus becoming a very difficult task to control at all.

Before presenting the most recent tools used in fault detection during knitwear production, it seems interesting to introduce the concept of weft knitting. This introduction will allow the reader to better understand the problem that is intended to be solved. Considering Fig. 1 and a circular knitting machine, it seems quite easy to understand how the weft knitted fabric is produced. One of the main characteristics

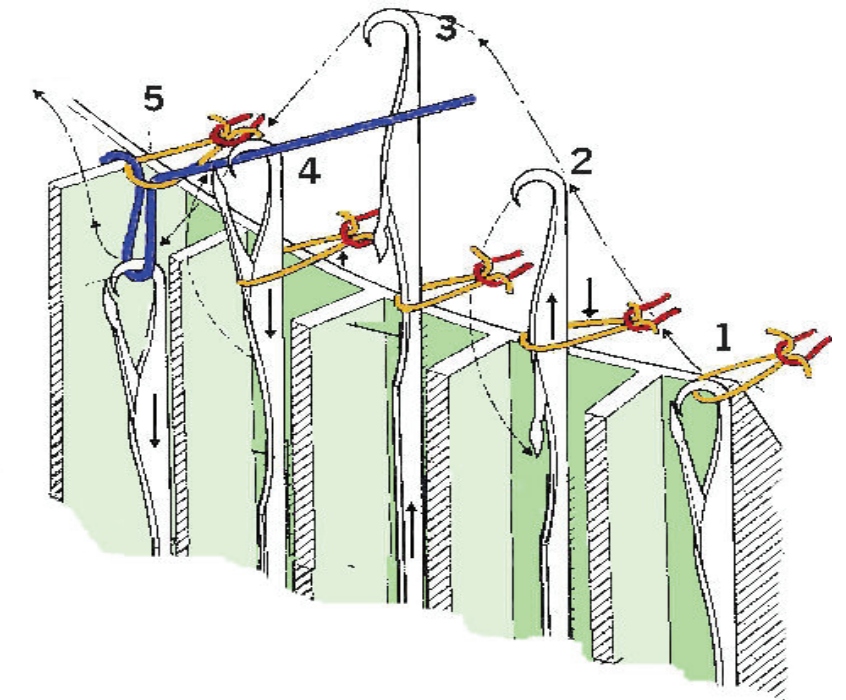

Fig. 1. Loop formation detail on weft knitting. As needles go upwards with the old loop sliding down ( 2 and 3 ) into the stem, the new yarn is fed until it reaches the hook (4); meanwhile the ltach is forced to close by means of the old loop and in (5 ) a new loop is formed, with the needle again in rest.

is that all what is needed to produce weft knitwear is a single yarn and a bed or cylinder with needles. The process it is not so simple, but is enough to illustrate the process [2]. Since one yarn is enough, it is also simple to understand that if the yarn breaks and the machine continues to work, the knitwear will fall. For that reason, the avoidance of sudden stops start immediately at the yarn reel, where sensors and actuators prevent yarn breaks to go further and promote the falling of the knitwear. The monitoring continues at the feeding stage, where the feeders are equipped with an intermediate yarn storage and yarn break detectors, before and after the storage subsystem. At the knitting cylinder, sensors detect needle failures or malfunctions, and finally, after the produced knitwear, optical devices detect faults, such as holes, stripes and other problems [4-8].

Nevertheless, the faults still appear, so further monitoring is still required.

\section{A. Fault Detection through Vision Techniques}

By far, the most used approach for detecting faults is made after the production of the knitwear. To this time consuming operation is called fabric inspection. The product under observation continuously moves by means of a roller system and a specified light environment allow the technician to carefully look for any fault present and still undetected after 
production. However, it is known that the operator's efficiency for detecting faults degrades with time due to human fatigue [9]. Nevertheless, vision is the approach chosen by almost all researchers in this area and several works have been developed, mainly on weaving and using many different techniques: neural networks (back propagation NN), Fuzzy Logic, Co-occurrence matrix, cluster analysis, etc. These techniques are used alone or combined in order to produce better results, achieving $90 \%$ of success in detecting and distinguishing faults in more than one publication [10-16]. All these proposals make use of CCD cameras or scanners to obtain the images, pre-process the signals and then apply the techniques. The biggest disadvantage on this approach is the fact that it requires a significant processing effort to the computer unit, thus reducing its capability for fast detection. The same problem arises when optical devices are used during production, since the processed image can't cope with the machines top speeds and random faults.

\section{B. Fault Detection through YIT}

Investigations started some years ago, in the sequence of one paper written by Wray et al [17]., where it is stated that the electrical signal resulting from the sensors installed in the stitch cam could be used to detect faults. It was then proposed an approach for detecting faults by monitoring the YIT. This parameter is the resulting force $(\mathrm{cN})$ of the needle's descending movement in order to produce a new loop (refer to Fig 1.). The effect produced in this force resembles a sinusoidal waveform, were the significant harmonic matches with the number of needles inside the cylinder. Many experiments were made and it becomes evident that this approach has much more advantages than the others, since if reflects exactly what is happening during the knitting process, and any abnormality would then be reported by an unusual waveform. Other reference paper states that the concept of CAQ - Computer Aided Quality Management should include all the information possible to gather in the knitting machine, which suits perfectly on the proposed approach [18]. The studies made have shown that is possible to detect all faults, locate them with an excellent accuracy and precision. In fact, the accuracy is of one to three needles and the precision is of one needle. These figures are quite impressive when considering knitting machines with 4000 needles, and achieving speeds of about 2000 needles per second. Moreover, further investigations have shown that it seems possible to distinguish the faults using for example cluster analysis [19]. So it seems evident that this approach must be considered as a valid tool for fault detection, since it allows detecting the faults during the loop formation, and not after the knitting formation, as it happens with the solutions provided so far by the main manufacturers [20]. This results in a significant saving in defective product. However, this approach demands one sensor for each yarn used, and for that reason a low cost solution was suggested [21], which would allow the assembling in all yarns used in a knitting machine. The next step is to investigate the possibility of automatically distinguish the faults and so identify the cause of the fault. Such achievement would dramatically decrease the stopping times for repair and at the same time, save investments made in changing the entire set of needles, when the technician detects one fault but he does not know what and where is the cause of the fault.

\section{GATHERING THE INFORMATION}

In this section the process of gathering the data for signal analysis will be briefly described, since it is not the ultimate goal of the present paper. Further information can be found in [22-23]. However, it is worth to mention that more improvements were made in the acquisition system, being at the present moment fully software programmable.

The core of the data gathering is the force sensor (for YIT acquisition and based on a complete bridge of strain gages), the encoders (responsible for synchronizing and data sampling), the pre-processing stage (software programmable instrument amplifiers, anti-aliasing filters and proper buffering), and the software application itself. The application was developed with LabVIEW® $6 . i$ and it is organized in several modules [22], with particular highlight on KnitLAB $\odot$ - the main application, and MonitorKNIT॰ [3]. The latter is responsible to detect the presence of faults and abnormalities during the knitting process, as it was previously described on Catarino et al [23]. This application has several stages that analyze thoroughly the YIT waveform as it is acquired, thus performing an on-line surveillance. When something considered by the decision module is not normal, decides if the problem is severe enough to stop the machine or simply acknowledge the main application of a potential problem. If the problem is really serious, the machine immediately stops and the gathered data, in proprietary form in transferred into the main application that will decide if it is a false alarm or a fault, indicating at the same time where it has occurred.

This is a brief summary of the entire system, with the only purpose of clearing the way the information is gathered.

\section{FAult Database}

The main purpose of this paper is to show the experiments made in order to prove that it would be possible to automatically distinguish faults by using multivariate statistics. First of all, it is important to mention that only time domain is considered in this paper, although frequency domain was also explored. The other important note is how the fault database was created. The procedure was quite clear: the faults were deliberately provoked before production of the knitwear, and selected depending on their appearance rate characteristics during production. The selection criteria were based on knitwear technician experience and also the characteristics of the knitting machine used for the experiments. It was used a sample circular weft knitting machine that uses only one yarn 
to produce knitwear, which constitutes an optimum environment for testing. The machine can work from $0.5 \mathrm{~m} / \mathrm{s}$ up to $2.0 \mathrm{~m} / \mathrm{s}$, and has 168 needles and the same number of retention sinkers.

The application gathered the data and stored it for further analysis and processing before applying the multivariate statistics techniques, thus building the fault database.

TABLE I

FAULTS USED TO FORM THE DATABASE

\begin{tabular}{|c|c|}
\hline Name & Color in graphics \\
\hline Needle missing & Green \\
\hline Needle without hook & White \\
\hline YIT with no Faults (control purposes) & Light Blue \\
\hline Needle without Latch & Orange \\
\hline Sinker missing & Yellow \\
\hline Needle with damaged Latch & Purple \\
\hline
\end{tabular}

\section{THE YIT WAVEFORM}

The following figures will show the typical waveform of YIT for the simplest weft structure: Jersey, where all needles will form a loop (as Fig. 1 represents). Fig. 2. shows an entire rotation of the knitting machine needle's cylinder. Each complete rotation corresponds to a course of loops. In the same figure are represented the faults experimented for a speed of $0.15 \mathrm{~m} / \mathrm{s}$. As it can be seen, there are differences that are more obvious in Fig 3. The yarn used is polyester continuous filament 240 dtex (tex is a S.I. unit and represents $\mathrm{g} / \mathrm{km}$ ). More experiments were also made with cotton yarn with a linear density of 24 tex.

Fig. 2 and Fig. 3 are very clear, because the waveforms were submitted to synchronization average. This technique, although very simple, allows the removal of a significant part of noise in the signal and at the same time pinpoints particular behaviours that could be interpreted as random. In this particular case, a fault that occurs in every complete rotation could pass without

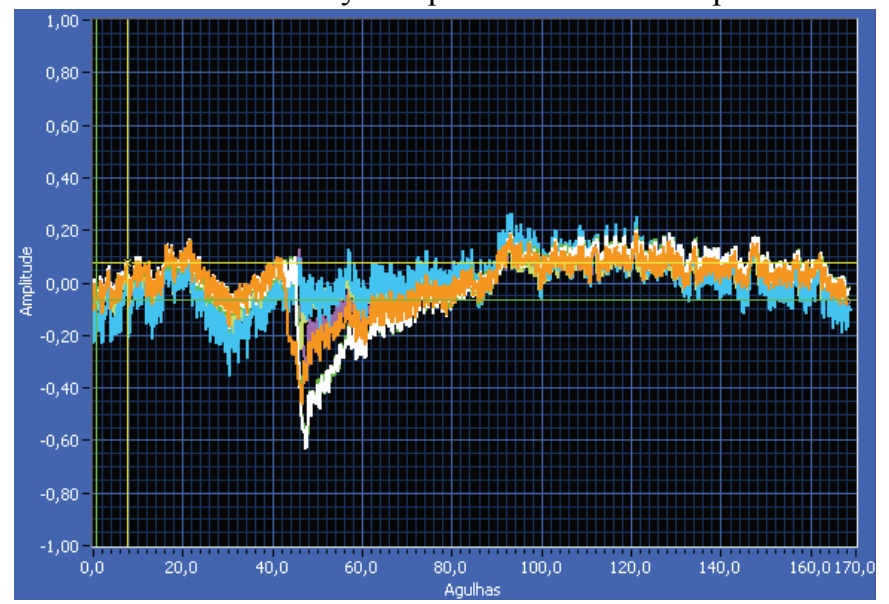

Fig. 2. Example with several YIT waveforms. All of them were average synchronized before visual representation. The vertical axis is represented in $\mathrm{V}$ and the horizontal axis was already transformed for needle position.

being noticed, but with this tool the problem would be enhanced.

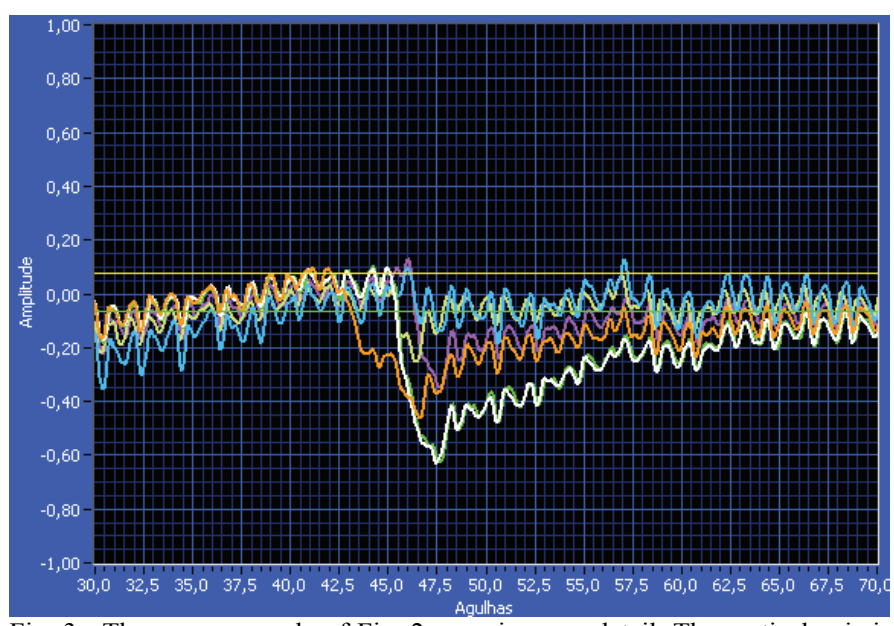

Fig. 3. The same example of Fig. 2, now in more detail. The vertical axis is represented in $\mathrm{V}$ and the horizontal axis was already transformed for needle position.

The average synchronization applied is quite simple to understand: consider each acquired point for $\mathrm{t}$ instant, or 1 space position, or even $\alpha$ angle as making part of a random variable $\boldsymbol{X}_{\mathrm{i}}$, where i will correspond to rotation i, acquired by the MonitorKNIT. The synchronized average YIT would be represented by a $\boldsymbol{T} 2 \mathrm{D}$ array, were the rotations (or courses) are represented from 1 to $\mathrm{m}$, and each acquired point for a

$$
T_{m, n}=\left(\begin{array}{ccc}
x_{11} & \ldots & x_{1 n} \\
\vdots & \ddots & \vdots \\
x_{m 1} & \cdots & x_{m n}
\end{array}\right)
$$

particular rotation (course) would be represented by 1 to $n$.

Each column represents one acquired sample of the YIT for one particular rotation. For example, $x_{11}$ would be the first

$$
T_{1}=\left[\begin{array}{c}
x_{11} \\
\vdots \\
x_{m 1}
\end{array}\right]
$$

acquired point for the first rotation (course). So, $\mathbf{T}_{\mathbf{1}, \mathrm{k}}$ represents the first wale of the knitted fabric.

Now, if one takes the arithmetic average of $\mathbf{T}_{1}$ and then do the same thing from 1 to $\mathrm{n}$ will have the synchronized average

$$
\begin{gathered}
\bar{T}_{1}=\frac{1}{m} \sum_{i=1}^{m} x_{i, 1} \\
\bar{T}_{\text {Synchronized }}=\left[\begin{array}{lll}
\bar{T}_{1} & \cdots & \bar{T}_{n}
\end{array}\right]
\end{gathered}
$$

of YIT:

As an illustration of the advantage of this tool, Fig. 4 shows the variability for the same yarn, in the very same conditions. This is the problem that one will have to face in order to create rules capable of automatically distinguish faults or even false 
alarms. It was mentioned earlier in this paper that the first problem concerning the identification of the presence of a fault is already treated [23]. The problem now is, if one can observe differences between faults, why can't do it automatically? This

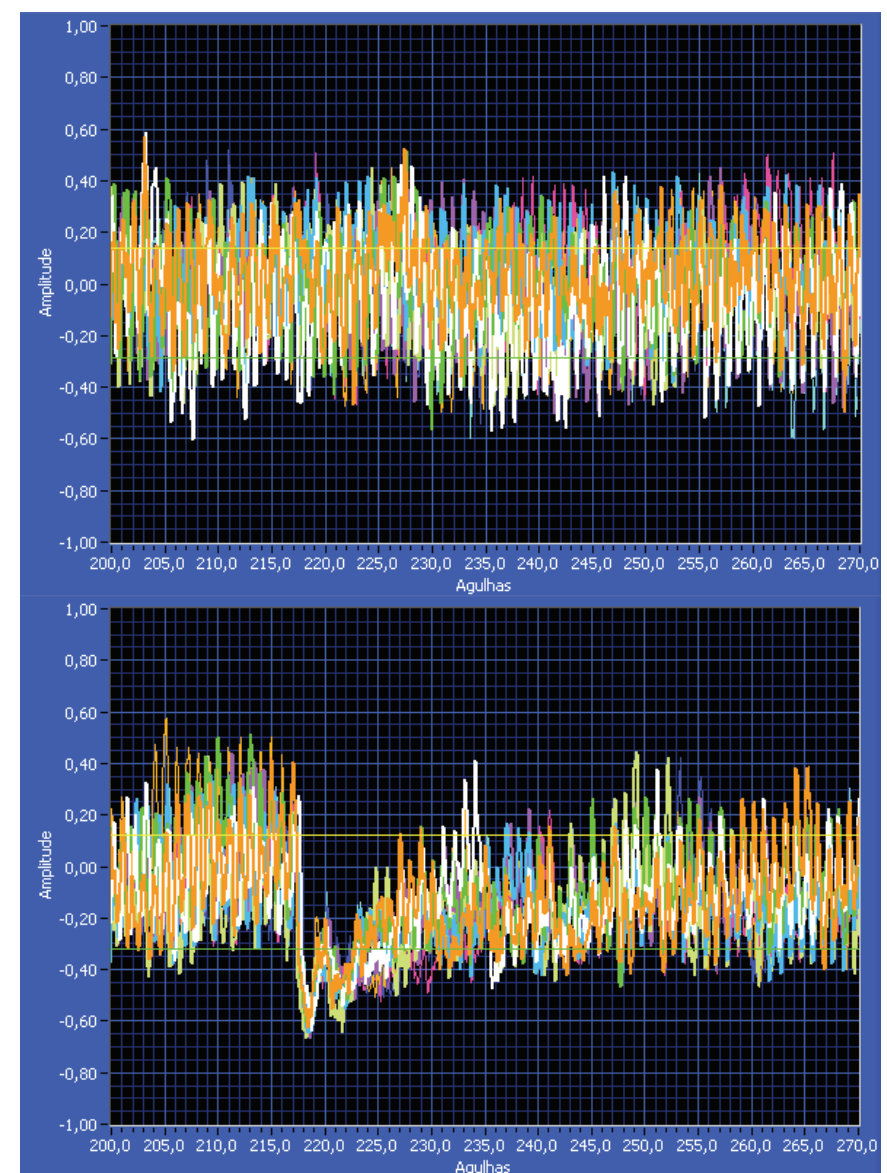

Fig. 4. Two illustrations for 10 consecutive courses/rotations for the same yarn (cotton 24 tex) in two different situations: on top no fault was present; below two needles are missing. The images were taken from an industrial knitting machine at $0.67 \mathrm{~m} / \mathrm{s}$. The vertical axis is represented in $\mathrm{V}$ and the horizontal axis was already transformed for needle position.

is the question that will be addressed in the following sections.

\section{Classifying Faults}

The first step on trying to prove that this approach is valid was to evaluate the capability of a multivariate statistical method would correctly organize unsorted cases in groups with similar characteristics inside, but different for the other groups. For this task, Cluster Analysis was selected. Hierarquical method was selected, and the agglomeration technique was chosen to be Ward's technique, since it allows the clustering through variance minimization. For the measure of proximity between clusters and cases, Euclidian Distance was chosen. The procedure of clustering analysis is rather big and it can be found in several references [25,26]. It seems however more important to describe how the YIT waveform was used in order to obtain results from Cluster Analysis.

Consider the matrix $\mathbf{T}_{\mathbf{m}, \mathbf{n}}$ presented in (1). Consider now column array $\mathbf{T}_{\mathbf{j}, \mathbf{1}}$, as expressed in (2). As it was already stated, this column represents one acquisition point during for each rotation stored. For the case of this sample knitting machine, 2000 points were acquired in each rotation (course). Considering that there are 168 needles, and each one produce one loop, one can say that each loop is approximately represented by 12 points. It is the same to say that $12 \mathbf{T}_{\mathbf{j}, \mathbf{i}}$ represent one wale of the knitted fabric. So, for the purpose of experimenting Cluster Analysis, a frame of $\mathrm{k}$ loops were subjected to the algorithm and the results analyzed, were $\mathrm{k}=$ $\{5, \ldots, 11\}$. Note that from the statistical point of view each column array $\mathbf{T}_{\mathbf{j}, \mathbf{i}}$ represents a random variable, with a normal distribution. At the final, the frames comprehend between 60 up to 132 variables. The cases considered for testing the Cluster Analysis are represented by the $j$ index on the $\mathbf{T}_{\mathbf{j}, \mathbf{i}}$ matrix. 50 rotations, or $\mathrm{j}=\{1, \ldots, 50\}$ were considered for each fault represented in Table I. To make sure that there was no influence on the waveform due to faults produced in different space positions, all faults were provoked in the same place and the frame extracted at the same vicinity.

Another important condition was the number of clusters that would be expected. From Table I, six clusters are expected; however, since two of the faults are quite similar (as Fig. 3 shows) it is possible that the only five will be correctly formed. So, the software (SPSS $\AA$ ) was instructed to produce solutions both for five and six clusters formed. Finally, three different situations of raw material were also considered. The total number of experiments was then 42. The following graphics summarize the results obtained.

From the results obtained it seemed evident that the five cluster solution (Fig. 5) is the best one, since the succeeding rate of correct grouping is maximized. The reason is closely related with the similarity of two different kinds of faults that result in very similar waveforms. The close inspection of Fig. 6 allows concluding that a frame of five loops is enough to obtain the best results possible. However, the cotton yarn reveals a very stable behavior, in contrast with polyester waveforms.

On the other hand, Fig. 6 clearly shows the uncertainty that the two particular cases (needle missing and needle without hook) produce when six clusters are required to be formed. However is important to note that for a more significant

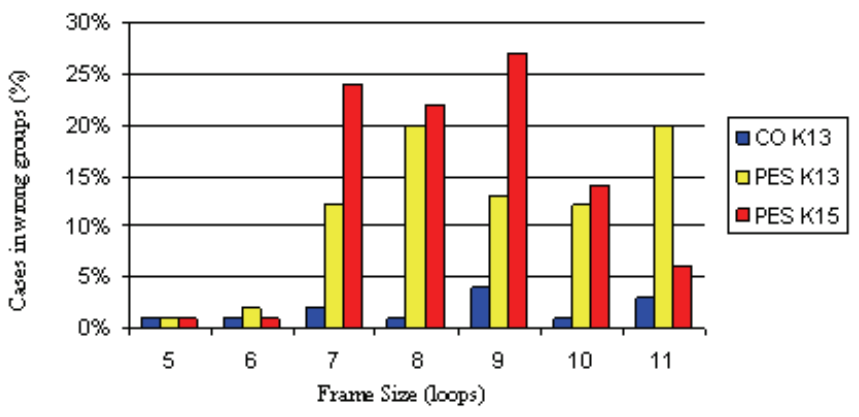

Fig. 5. Graphical representation of the cases grouped in the wrong cluster, for the case of 5 clusters extracted. The three situations involved polyester (PES) c.f. yarn for tightness factor 13 and 15, and cotton (CO) yarn 24 tex with a tightness factor of 13 . 


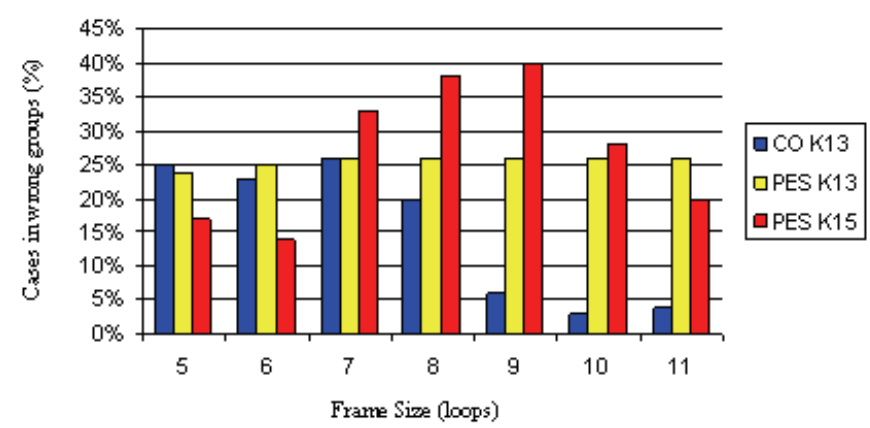

Fig. 6. Graphical representation of the cases grouped in the wrong cluster, for the case of 6 clusters extracted. The three situations involved polyester c.f. yarn for tightness factor 13 and 15, and cotton yarn 24 tex with a tightness factor of 13 .

number of loops in the frame, Cluster Analysis is able to correctly form the six clusters, but only for cotton yarn. This is rather curious, since cotton is the yarn with more severe variability in each rotation due to its physical properties, namely the yarn evenness [3]. There are also one or two training cases that were wrongly assumed as false alarms (no fault present) which could be critical. The reason maybe related with the random selection of the submitted cases. A careful selection would probably avoid this situation.

Concluding, the five clusters solution is a good result, since two faults are so similar that even the authors could not correctly judge the cause of the fault, if the waveforms were given to them without any information. Nevertheless, what is worth to mention is the capability of Cluster Analysis to correctly group the situations, and, in the case of Fig. 7, was even capable to distinguish the two similar situations, which is quite remarkable. This proves that this tool can be used with success with the YIT approach.

\section{Discriminating CAUSES OF FAUlts}

The next step was to establish rules for discriminate and correctly judge submitted cases after a fault detection made by MonitorKNIT in on-line surveillance. With this aim, another Multivariate Statistical technique was chosen, namely, Discriminant Analysis. Basically, this technique create rules, known as canonical equations or functions, that will give the capability to classify a newer case in one of the groups previously created with the help of training cases.

Since it was concluded from the previous section that five loops ( $5 \times 12$ columns) would be enough to correctly group the training cases, this number, together with another column, called as "cluster group number" were fed into the Discriminant Analysis algorithm. This column can be supplied directly from the Cluster Analysis results or manually. In this particular experiment manual approach was used in order to force the algorithm to generate the rules for the six cases of Table I. Another important condition was that, from the set cases used for training (300 cases), only $80 \%$ was really used for training the model. The remaining $20 \%$ were fed into the resulting model in order to evaluate its performance when an unknown case is submitted into the obtained model. The selection of the cases was once more completely random. No

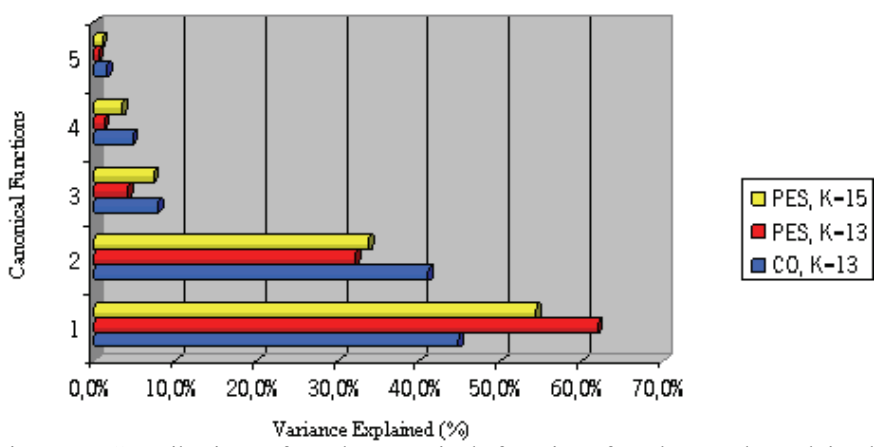

Fig. 7. Contribution of each canonical function for the total explained variance. The three situations involved polyester c.f. yarn for tightness factor 13 and 15, and cotton yarn 24 tex with a tightness factor of 13.

further processing was used besides the preprocessing stages and normalization used in this tool.

Fig. 7 shows a similar behavior for the three kinds of yarn and tuning, where the first two canonical functions contribute for almost all variance. In fact, the close inspection of Table II allows concluding that three canonical functions would explain more than $94 \%$ of the global variance, and four canonical functions explain about $99 \%$. However all functions were used and the most important figure in Table II is the fourth line, where the $20 \%$ cases not used to obtain the canonical functions are submitted and classified. The table shows that cotton presents an impressive success rate - 98.3\%, never misclassifying any case with false alarms (not visible in this table). The same has happened with the other two situations of polyester, although the success rate has decreased about $10 \%$. The reason for this relative unsuccessful result is again the similarity of the previously mentioned situations. However, in cotton was capable to distinguish them. It seems that if a five cluster column was used directly from the Cluster Analysis results the success would be improved, since this ambiguity would disappear.

TABLE II

RESULT TABLE FOR DISCRIMINANT ANALYSIS APPLIED TO CASES

\begin{tabular}{|c|c|c|c|}
\cline { 2 - 4 } \multicolumn{1}{c|}{} & $\begin{array}{c}\text { POLYESTER, } \\
\mathrm{K}=13\end{array}$ & $\begin{array}{c}\text { POLYESTER, } \\
\mathrm{K}=15\end{array}$ & $\begin{array}{c}\text { COTTON, } \\
\mathrm{K}=13\end{array}$ \\
\hline $\begin{array}{c}\text { \% EXPLAINED VARIANCE USING 4 CANONICAL } \\
\text { FUNCTIONS }\end{array}$ & $\mathbf{9 9 . 5}$ & $\mathbf{9 9 . 0}$ & $\mathbf{9 8 . 5}$ \\
\hline $\begin{array}{c}\text { \% EXPLAINED VARIANCE USING 3 CANONICAL } \\
\text { FUNCTIONS }\end{array}$ & $\mathbf{9 8 . 3}$ & $\mathbf{9 5 . 5}$ & $\mathbf{9 3 . 8}$ \\
\hline $\begin{array}{c}\text { \% CASES ORIGINALLY SELECTED AND } \\
\text { CORRECTLY CLASSIFIED WITH FULL MODEL }\end{array}$ & $\mathbf{9 8 . 3}$ & $\mathbf{9 9 . 2}$ & $\mathbf{9 6 . 2}$ \\
\hline $\begin{array}{c}\text { \% CASES ORIGINALLY NOT SELECTED AND } \\
\text { CORRECTLY CLASSIFIED WITH FULL MODEL }\end{array}$ & $\mathbf{8 8 . 1}$ & $\mathbf{8 8 . 1}$ & $\mathbf{9 8 . 3}$ \\
\hline $\begin{array}{c}\text { \% CASES SEPARATELY SELECTED AND } \\
\text { CORRECTLY CLASSIFIED WITH FULL MODEL }\end{array}$ & $\mathbf{9 2 . 0}$ & $\mathbf{9 3 . 3}$ & $\mathbf{9 3 . 8}$ \\
\hline
\end{tabular}

\section{DISCUSSION}

Since Cluster Analysis has given such promising results why shouldn't be included in the process, by supplying the array that classifies the learning cases for the Discriminant Analysis? 
With this tool, the system would be able to relearn new situations, which is one major request for a reliable pattern recognition system. This will be one of the next stages in this

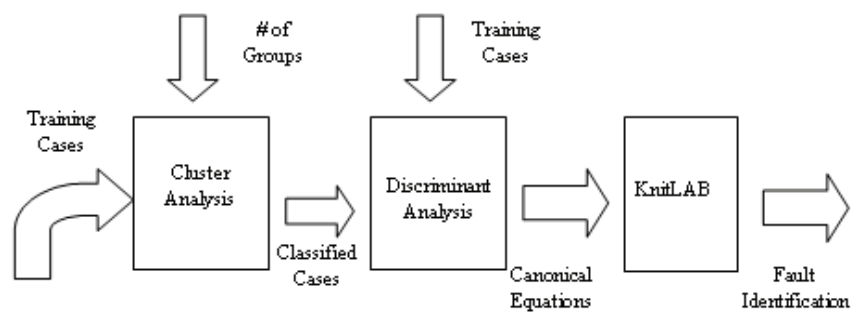

Fig. 8. General structure overview for the experiments made with Multivariate Statistics.

project, which is already in progress with the structure suggested in Fig. 8.

It is evident from the results obtained that the Multivariate Statistical tools selected can in fact automatically distinguish the faults, even when the similarity is quite relevant. Together they can supply the canonical function arrays for the main application KnitLAB and, besides detecting and locating faults, distinguish the cause, thus contributing for higher quality in product and even more increased productivity.

More research continues to be developed with the purpose of improving this approach as well as the software. Different yarns are being tested at higher speeds and other faults are being added into the database. At the same time industrial knitting machines are starting to be used with very promising results.

\section{CONCLUSIONS}

This paper presented, together with an approach with high potential for fault detection in weft knitting - the yarn input tension, the application of Multivariate Statistical tools for classification of the cause of faults generated during the production of knitwear. At the same time has also shown that there are other alternatives, although not so new than the techniques more recently used for classifying faults that are also capable to have similar or even better results.

\section{ACKNOWLEDGMENT}

The authors wish to thank the sponsors of this research project, FCT - Fundação para Ciência e Tecnologia, project POSI/SRI/39824/2001 and the University of Minho $\left({ }^{1,2}\right.$ Textile Engineering Department and ${ }^{3,4}$ Electronics Engineering Department).

\section{REFERENCES}

[1] J.M. Juran, F.M. Gryna, Quality Planning and Analysis - From Product Development through Use, $2^{\text {nd }}$ ed., TATA McGraw-Hill, New Delhi, 1982

[2] D.J. Spencer, Knitting Technology - A Comprehensive Handbook and Practical Guide, $3^{\text {rd }}$ ed., Woodhead Publishing Limited, Pergamon Press, Cambridge: England, 2001, ISBN 1855733331

[3] A. Catarino, Monitorização e Controlo em Tempo Real do Processo de Tricotagem em Teares de Malha de Trama, PhD Thesis, University of Minho, 2005
[4] Needle Detector NW from Memminger-IRO , reference 335.905.001.07 DSNL/5, official website http://www.memminger-iro.de/ (30-07-2005).

[5] Digital Needle Sensor Type 4020, Protechna Herbst GmbH \& Co. KG, official website http://www.protechna.de/e/nadtast d.htm (30-07-2005).

[6] Fabric Scanner LMW 3, Memminger-IRO, reference 040.905.000.01 DUDD/40, official website http://www.memminger-iro.de/ (30-07-2005).

[7] Yarn detectors OFW/UFW, Memminger-IRO, official website http://www.memminger-iro.de/ (30-07-2005)

[8] H. Behr, "Accessories for circular and flat knitting machines", Knitting Technology, 1996, Vol. 18, 4, pp. 184-189

[9] M. Bradshaw, "The Application of Machine Vision to the Automatic Inspection of Knitted Fabrics", Mechatronics, 1995, 5, pp. 233-243

[10] Xu, Bugao, "Identifying Fabric Structures with Fast Fourier Transform Techniques", Textile Research Journal, 1996, 66, pp. 496-506

[11] M. Srdjak, Z. Vrljičak, "Applying Discrete Fourier Transform to the Knitting Process", Textile Research Journal, 2001, 71, pp. 384-387

[12] Yau-Ren Shiau, I-Shou Tsai, Chih-Shiang Lin, "Classifying Web Defects with a Back-Propagation Neural Network by Colour Image Processing", Textile Research Journal, 2000, 70, pp. 633-640

[13] M.C. Hu, I.S. Tsai, "Fabric Inspection Based on Best Wavelet Packet Bases", Textile Research Journal, 2000, 70, pp. 662-670

[14] B. Mallik-Goswami, A. K. Datta, "Detecting Defects in fabric with Laser-Based Morphological Image Processing", Textile Research Journal, 2000, 70, pp. 758-762

[15] Chang-Chiun Huang, I-Chun Chen, "Neural-Fuzzy Classification for Fabric Defects", Textile Research Journal, 2001, 71, pp. 220-224

[16] Hyung Taek Choi, Sung Hoon Jeong, Sook Rae Kim, Jae Yun Jaung, Seong Hun Kim, "Detecting Fabrics with computer Vision and Fuzzy Rule Generation - Part I: Defect Identification by a Fuzzy Expert System", Textile Research Journal, 2001, 71, pp. 563-573

[17] G.R. Wray, N.D. Burns, "Cam-to-Needle Impact Forces in WeftKnitting, Part IX, X and XI", Journal of the Textile Institute, 1978, vol. 69, pp. 301-315

[18] G. Buhler, L. Pestel, H. Hait, "Requirements in regard to future circular knitting assurance systems- Practical trial with a future-oriented monitoring system", Melliand textilberichte, 1, 1994, p 39-42

[19] A. Catarino, A. M. Rocha, J. Monteiro; "Automatic Fault Identification in Knitting Machines", in Proceedings of Signal Processing, Pattern Recognition and Applications 2001 IASTED International Conference, pp. $5-10$

[20] A. Catarino, A. Rocha, J. Monteiro, "Monitoring Knitting Process through Yarn Input Tension: New Developments", IEEE proceedings of IECON 2002, Vol. III, pg 2022-2028, ISBN 0-7803-7475-4

[21] A. Catarino, A. Rocha, J. Monteiro, "Low Cost Sensor for the Measurement of Yarn Input Tension on Knitting Machines", in proceedings of the ISIE 2003 - International Symposium on Industrial Electronics, ISBN 0-7803-7912-8, 2003

[22] Catarino A., Rocha A., Monteiro J.L., Soares F., "A New System for Monitoring and Analysis of the Knitting Process", in proceedings of the ICM'04 - IEEE International Conference on Mechatronics 2004, ISBN 0-7803-8600-0, Istambul, Turkey, June 2004

[23] Catarino A., Rocha A., Monteiro J. L., Soares F., "Techniques for Unveiling Faults During Knitting Production", in proceedings of IEEE ISIE 2004 - IEEE International Symposium on Industrial Electronics, ISBN 0-7803-8305-2, Ajaccio, France, May 4-7, 2004

[24] A. Catarino, A. Rocha, J.L. Monteiro and F. Soares, "Knitting Process Surveillance Using Time and Frequency Analysis", IEEE ISIE 2005, Dubrovnik, Croatia, ISBN:0-7803-8739-2, pp. 1563-1568, June 20-23, 2005

[25] S. Landau, B. S. Everitt, A Handbook of Statistical Analyses using SPSS CHAPMAN \& HALL/CRC, A CRC Press Company, Chapman \& Hall/CRC Press LLC, ISBN 1-58488-369-3, Boca Raton, Florida, U.S., 2004

[26] SPSS ${ }^{\circledR} 13.0$ Base User's Guide, Copyright (C) 2004 by SPSS Inc, Chicago, U.S. 